\title{
EDITORIAL \\ Emerging zoonoses: tackling the challenges
}

It is widely accepted that most emerging diseases are zoonotic, i.e. naturally transmitted between vertebrate animals and humans, often involving complex transmission pathways that may also include arthropods $[1,2]$. In recent years, emerging zoonoses have received increasing attention due to major outbreaks such as SARS and Ebola.

According to the European Food Safety Agency (EFSA), an emerging risk is defined as 'a risk resulting from a newly identified hazard to which significant exposure may occur or from an unexpected new or increased significant exposure and/or susceptibility to a known hazard'. Thus, emerging zoonoses may be caused by previously unknown agents (i.e. 'true' emergence) or by changes in the exposure or susceptibility of hosts leading to an increased number of cases. Some authors also distinguish between global emergence-outbreaks due to agents observed for the first time worldwide, or local emergence - occurrence of a known agent in geographical areas previously free of disease. There may also be apparent disease emergence caused by increased disease awareness or improved or new diagnostic tools. The latter should be distinguished from true emergence [3].

Numerous factors have been identified as drivers of disease emergence [4-7]. These include both characteristics of the pathogen itself such as high mutation rate in RNA viruses, as well as characteristics of the host such as human demographics and behaviour. The latter can include, for example, changes in dietary habits or behaviour which can increase the relevance of previously rare exposure pathways. More frequent travelling to exotic places or consumption of exotic foods such as bush meat are examples of such changed behaviour. Moreover, through an increase in air passenger numbers, pathogen incursions via human movement can become a real risk [8]. Finally, ecological and environmental changes can also lead to changes in the distribution of agents or their vectors [7]. Although inextricably linked to the factors above, climate change is a major driver for disease emergence. There are already several examples where changes in temperature and/or rainfall have led to the spread of zoonotic diseases. In Europe, this is true for West Nile virus, dengue virus and chikungunya virus. Flooding can be an additional risk factor. Finally, changes in agricultural practices and particularly global trade can lead to an increased probability of spread of agents [9]. The understanding of these driving factors is therefore central to preparedness. A key lesson learnt on emerging diseases is that we need to understand and - if possible - anticipate the convergence of factors related to the characteristics of the microbe and host, as well as their complex interaction simultaneously or in sequence [10].

Despite ongoing research, the epidemiology of many zoonoses remains obscure. Even for pathogens that have been known for a long time in humans, the animal reservoir can remain vaguely defined or unknown. This involves not only diseases causing minor disease burden such as tularaemia where pathogenic strains are rare $[3,11]$, but also major public health threats such as Ebola. Certain animals are mentioned in the context of several emerging diseases, most notably a number of bats (Chiroptera) which have been associated with disease outbreaks caused by both Hendra and Nipah viruses. Epidemiological and genomic analyses suggest a single zoonotic transmission event from a bat to a 2-year old boy was the initiating factor for the recent Ebola outbreak in West Africa $[12,13]$. Human behaviour then became the key driver for the subsequent human-to-human transmission which led to a major outbreak.

In order to be able to more effectively manage outbreaks of emerging zoonoses, early warning systems are being developed. Such systems are widely used and a range of methods are available [14]. Nevertheless, to achieve mitigation of emerging zoonoses, it is important to integrate surveillance into a wider context of general preparedness and rapid response. This should be 
achieved at a time when resources for both veterinary and public health services are being reduced. Prioritization is thus essential to use limited resources in an effective way. A range of approaches to priority setting is in use [15], but a better integration and strategic use is warranted.

Methods such as risk assessment or simulation modelling can also be applied to estimate the probability of pathogen emergence, incursions into previously free areas and subsequent spread [13, 15]. However, as we are dealing with novel risks, there will be major uncertainties and paucity of data underpinning these models. In such situations, expert opinion may be the only option. Limitations of this approach are well understood and measures to assure use of good practice should be taken. To include more experts is not always a solution as they may have diverging views the combination of which can cause further challenge [16].

Emerging zoonoses are often complex in their biology and epidemiology. Their effective control therefore requires a combination of knowledge and skills across disciplines. The need for collaboration involving public health specialists and veterinarians, and also environmental scientists, economists, sociologists and anthropologists has been repeatedly emphasized $[11,13,15]$. Such an approach would follow the so-called One Health paradigm. There are many definitions of One Health, and they generally cover efforts to provide more effective, evidence and systems-based health interventions. Examples of implementations of the One Health concept are unfortunately still rare. A recent international round-table discussion called for more inter-related training of medical, veterinary and environmental science students to create conditions in which One Health would offer the obvious approach [17]. This would be particularly useful for the control of zoonoses and essential for the early detection and effective management of disease emergence. The outbreak of Ebola that occurred across several countries in West Africa provides a stark example of the consequences of delayed and inefficient response. It also creates an opportunity to investigate influential factors for successful and rapid management of disease emergence. Priorities for capacity-building for the control of emerging zoonotic diseases have recently been identified [18]. Efforts should include the development of risk management policies, human and diagnostic capacity building as well as cost-benefit considerations for setting priorities when resources are limited.

\section{REFERENCES}

1. Cleaveland S, Laurenson MK, Taylor LH. Diseases of humans and their domestic mammals: pathogen characteristics, host range and the risk of emergence. Philosophical Transactions of the Royal Society of London, Series B: Biological Sciences 2001; 356: 991-999.

2. Cutler SJ, Fooks AR, van der Poel WHM. Public health threat of new, reemerging, and neglected zoonoses in the industrialized world. Emerging Infectious Diseases 2010; 16: $1-7$.

3. D'Alessandro D, et al. Human tularemia in Italy. Is it a re-emerging disease? Epidemiology and Infection. Published online: 22 October 2014. doi:10.1017/ S0950268814002799.

4. Morse SS. Factors in the emergence of infectious diseases. Emerging Infectious Diseases 1995; 1: 7-15.

5. Anderson PK, et al. Emerging infectious diseases of plants: pathogen pollution, climate change and agrotechnology drivers. Trends in Ecology and Evolution 2004; 19: 535-544.

6. Jones KE, et al. Global trends in emerging infectious diseases. Nature 2008; 451: 990-993.

7. Weaver SC, Reisen WK. Present and future arboviral threats. Antiviral Research 2010; 85: 328-345.

8. Van Den Bossche D, et al. Chikungunya virus and West Nile virus infections imported into Belgium, 2007-2012. Epidemiology and Infection. Published online: 2 April 2014. doi:10.1017/S0950268814000685.

9. Wallace R, Wallace RG. Blowback: new formal perspectives on agriculturally driven pathogen evolution and spread. Epidemiology and Infection. Published online: 4 February 2014. doi.org/10.1017/S0950268814000077.

10. Smolinski MS, Hamburg MA, Lederberg J (eds). Microbial threats to health: emergence, detection, and response. Institute of Medicine (US) Committee on Emerging Microbial Threats to Health in the 21st Century. Washington, DC, The National Academies Press, 2003, pp. 397.

11. Hestvik G, et al. The status of tularemia in Europe in a one-health context: a review. Epidemiology and Infection. Published online: 30 September 2014. doi.org/10.1017/ S0950268814002398.

12. Baize S, et al. Emergence of Zaire Ebola virus disease in Guinea - preliminary report. New England Journal of Medicine 2014; 371: 1418-1425.

13. Alexander KA, et al. What factors might have led to the emergence of Ebola in West Africa? PLOS Neglected Tropical Diseases (in press). [preliminary version available online: http://blogs.plos.org/speakingofmedicine/2014/11/ 11/factors-might-led-emergence-ebola-west-africa/].

14. Rodríguez-Prieto V, et al. Systematic review of surveillance systems and methods for early detection of exotic, new and re-emerging diseases in animal populations. Epidemiology and Infection. Published online: 12 September 2014. doi.org/10.1017/S095026881400212X.

15. Brookes VJ, et al. Preparedness for emerging infectious diseases: pathways from anticipation to action. Epidemiology and Infection. Published online: 12 December 2014. doi.org/10.1017/S095026881400315X. 
16. Roche SE, et al. Assessing the risk of Nipah virus establishment in Australian flying-foxes. Epidemiology and Infection. Published online: 4 February 2014. doi.org/ 10.1017/S0950268813003336.

17. Stärk KDC, et al. One health surveillance - more than a buzz word? Preventive Veterinary Medicine. Published online: 11 February 2015. doi:10.1016/j. prevetmed.2015.01.019.

18. Gebreyes WA, et al. The global one health paradigm: challenges and opportunities for tackling infectious diseases at the human, animal, and environment interface in low-resource settings. PLoS Neglected Tropical Diseases 2014; 8: e3257.

\author{
K. D. C. STÄRK ${ }^{1}$ AND D. MORGAN ${ }^{2,3}$ \\ ${ }^{1}$ Royal Veterinary College, London, UK \\ ${ }^{2}$ Public Health England, London, UK \\ ${ }^{3}$ London School of Hygiene and Tropical Medicine, \\ London, UK \\ (Email: kstaerk@rvc.ac.uk)
}

